\title{
7 Conclusiones generales
}

La finalidad de este último capítulo es, esencialmente, comprobar que las cuatro preguntas planteadas al comienzo de esta investigación han quedado respondidas y recoger las ideas y resultados más destacados que han ido surgiendo a lo largo de todo el trabajo. Asimismo, se consideran los posibles caminos de investigación que quedan abiertos tras las aportaciones de este estudio, no solo acerca del MDO, sino a un nivel más amplio, en lo que a la conceptualización de los eventos o las relaciones de transitividad se refiere.

En primer lugar, por tanto, recuperaremos de nuevo las distintas preguntas que se establecieron al comienzo de la investigación:

- ¿Influye la telicidad del predicado en el uso del MDO?

- ¿Contribuye la afectación del objeto a la aparición de la marca preposicional?

$\mathrm{Y}$, en caso de que las primeras tuviesen una respuesta afirmativa, se planteaban otras dos preguntas más específicas:

- ¿El efecto de la telicidad/afectación se puede apreciar tan solo a nivel sincrónico o es posible documentarlo, asimismo, a nivel diacrónico?

- ¿Cómo se explica, desde un punto de vista lingüístico, que la telicidad y/o la afectación contribuyan al uso del MDO junto a otros factores como la animacidad o la definitud?

Para responder a las dos primeras preguntas, el capítulo 2 examinó desde un punto de vista sincrónico todos aquellos factores cuyo impacto en el MDO había sido señalado por la bibliografía previa. Dicha revisión del estado de la cuestión proporcionó la información suficiente para justificar la realización de la presente investigación y ayudó a delimitar cuáles eran los ámbitos que habían quedado más desatendidos hasta el momento. Mientras que los estudios previos habían analizado con gran profundidad la manera en que se producía el impacto de las características relativas al SN en el MDO, los factores dependientes del núcleo verbal no habían recibido ni mucho menos la misma atención. Concretamente, la telicidad del predicado y el grado de afectación del objeto directo constituían los factores de los que menos información se tenía, en lo que respectaba a cuál era realmente su impacto en el fenómeno y de qué forma se relacionaban con el resto de factores. Los escasos trabajos que habían abordado estos factores lo habían hecho, generalmente, a través de un número muy reducido de ejemplos basados en la introspección y, por lo tanto, no existían aún datos empíricos que respaldasen las afirmaciones realizadas al respecto. 
Partiendo de las certezas acerca de los factores nominales y como respuesta a las carencias encontradas en el capítulo 2, se llevó a cabo un cuestionario de elección forzada, presentado en el capítulo 4. Dicho cuestionario fue realizado con la finalidad de comprobar el efecto de la telicidad y la afectación en el MDO, siguiendo una metodología que hiciese posible fundamentar las conclusiones en datos empíricos. Para poder detectar el efecto de los factores, los objetos que se incluyeron en el estudio fueron los constituidos por SN indefinidos con referente humano, es decir, los que se encuentran en el ámbito de posibilidad del MDO en español. De esta manera, de producirse algún efecto, este sería el ámbito en el que podría hacerse visible. Por consiguiente, partiendo del cruce de factores, fueron creadas cuatro clases de contextos que respondían a los rasgos [ \pm télico] y [ \pm afectado], con el objetivo de poner a prueba tanto el efecto independiente de cada factor, como su posible interacción. El estudio permitió detectar un claro impacto de la afectación sobre el MDO que relaciona el uso de la marca con los objetos afectados; y, por otro lado, un ligero efecto de la telicidad que, en contra de lo previsto, relacionaría la aparición del marcado con los predicados atélicos. La metodología utilizada implica la ventaja de que los datos obtenidos son el producto de enfrentar a los participantes a contextos previamente controlados, lo que asegura que puedan excluirse determinados factores que sea necesario controlar. Por otra parte, el uso de cuestionarios también plantea limitaciones que, en este caso, se derivan también del mismo hecho de que el investigador es quien controla cada contexto, por lo tanto, los datos que se obtienen están influidos por el contexto en el que se recogen y se apartan quizá de aquellos que pueden encontrarse en el lenguaje espontáneo.

De forma paralela a la visión sincrónica ofrecida en estos capítulos, los capítulos 3 y 5 se han empleado para desarrollar la parte diacrónica de la investigación. El primero de ellos revisó los estudios más relevantes que han sido realizados a propósito de los factores que se analizaban de forma sincrónica en el capítulo 2. Este «complemento» teórico al segundo capítulo surge con la finalidad de constituir el punto de partida para poder responder a la tercera de las preguntas formuladas en la introducción. Teniendo en cuenta los resultados arrojados por el cuestionario, el siguiente paso era preguntarse si los efectos observados podían apreciarse también en etapas previas de la lengua. Con este propósito, se llevó a cabo un exhaustivo análisis diacrónico de corpus basado en los datos del CDH. Dicho análisis, presentado en el capítulo 5, ha examinado los datos extraídos de tres intervalos temporales diferentes que se corresponden con los siglos XIV, XVI y Xx. Además, para que los resultados fuesen comparables con los obtenidos en la prueba anterior, pero a la vez pudiese apreciarse con claridad la evolución del MDO, se incorporaron al estudio tanto SN léxicos definidos como indefinidos con referente humano. Los resultados de este análisis revelaron un 
marcado impacto de la afectación sobre el MDO en los SN indefinidos con referente humano. De la misma forma, mostraron una ligera tendencia, en la misma línea que el cuestionario, según la cual se apreciaba cómo los predicados atélicos favorecían ligeramente el uso de la marca preposicional frente a los predicados télicos. No obstante, esta ligera tendencia ni siquiera resultó ser significativa en este caso y, además, se pudo comprobar cómo, con mucha seguridad, era debida al comportamiento de determinados verbos y a diferentes factores que no habían sido controlados en el análisis, como la agentividad o las preferencias de selección del verbo. Sea como fuere, el aspecto más destacable de los resultados del análisis diacrónico es la marcada coherencia que mostraron con respecto a los obtenidos en el cuestionario, respaldando los datos de este y posibilitando su mejor comprensión al observar cuál había sido la evolución de los efectos.

En lo que respecta a la metodología utilizada, en este caso los datos se corresponden con oraciones extraídas de un discurso «cuasi libre», pues se trata de textos escritos. Este hecho permite encontrar ejemplos de MDO en contextos naturales, sin embargo, a diferencia de lo que ocurría en los cuestionarios, no es posible controlar todos los factores que determinan la aparición del marcado en cada caso y, por ello, los resultados pueden verse influidos en ocasiones por factores que escapan al control del investigador. Este hecho convierte al estudio mediante cuestionarios y al análisis de corpus en dos metodologías que se complementan bastante bien, si se tienen en cuenta las ventajas y limitaciones de una y otra. Por ello, la coherencia de los resultados de las dos pruebas realizadas cobra especial importancia al tratarse de dos metodologías tan distintas apuntando en una misma dirección.

Con todo ello, se puede afirmar que las tres primeras preguntas planteadas al inicio han quedado respondidas a lo largo de los cinco primeros capítulos. Quedaba así establecido que, mientras que la telicidad no ha demostrado haber ejercido una clara influencia directa en el MDO, la afectación se constituye como un factor que ha influido en el fenómeno desde el comienzo de su evolución hasta nuestros días. Su efecto interactúa con los factores nominales, siendo estos los que delimitan las zonas de marcado posible, imposible u obligatorio, y restringiendo el efecto de la afectación al dominio de posibilidad. Por lo tanto, la única cuestión que quedaba por plantearse era la que se preguntaba por el razonamiento lingüístico que podía existir detrás del impacto de la afectación en el MDO, documentado en las pruebas realizadas.

El planteamiento propuesto al respecto de esta cuarta pregunta se presenta en el capítulo 6. El enfoque que se propone considera el MDO, de forma paralela a la marca preposicional del dativo, como un marcador asociado a un conjunto de rasgos que, a su vez, son la causa de que el objeto en cuestión adquiera un elevado grado de prominencia. El origen de MDO tiene lugar a partir de un cambio que 
ya se había producido en latín previamente: la desaparición del morfema flexivo de dativo en favor del giro preposicional ad + acusativo. Dicha forma de marcado comenzó entonces a asociarse al conjunto de rasgos que caracterizaban a los dativos y, llegados a un punto, la misma marca comenzó a utilizarse para señalar determinados objetos directos que podían confundirse con los dativos debido a sus características. Entre los rasgos que definen al dativo, la bibliografía ha apuntado generalmente su carácter animado, definido y de tópico. Asimismo, el dativo presenta también ciertos rasgos agentivos que lo acercan al papel de Agente, y lo diferencian del Tema/Paciente. Por lo tanto, cuando los objetos directos presentaban esta serie de características comenzaron a recibir la misma marca que ya recibían los dativos.

Sin embargo, el análisis diacrónico llevado a cabo en esta investigación ha señalado que un rasgo cuyo efecto se ha podido observar desde el comienzo de la evolución del MDO es el carácter afectado de los objetos. De esta manera, cabría esperar que este factor estuviese en alguna medida relacionado también con los dativos. Esta postura es la que se ha defendido a lo largo de todo el capítulo 6, respaldando los resultados obtenidos en esta investigación con los distintos de trabajos que han argumentado en este sentido.

Para ello, ha sido necesario adoptar la definición de afectación basada en los trabajos de Jackendoff (1990) y Cruse (1973), fundamentados a su vez en el modelo de dinámica de fuerzas de Talmy (1976). Este enfoque conceptualiza los eventos en términos de transferencia de fuerzas y considera, por tanto, afectados a todos aquellos participantes, distintos del Agente/Actor, que son receptores de la fuerza que este genera. Desde esta perspectiva es posible defender que los primeros objetos directos en los que se originó el MDO no se caracterizaban solo por su animacidad y definitud, sino también por quedar afectados por la acción proyectada por el verbo.

La estrecha relación documentada desde los comienzos del fenómeno entre objetos directos con MDO y objetos indirectos o dativos, encuentra asimismo respaldo en los distintos estudios diacrónicos que han abordado el tema. Dichos estudios han apuntado una serie de cambios que avocan a la progresiva disolución de la distinción que existe entre estos dos tipos de argumentos. De hecho, según Company Company (2002a; 2003; 2006) estos cambios están propiciando que el español pase de ser una lengua de acusativo/dativo, a convertirse en una lengua que distingue entre objeto primario y objeto secundario.

Por todo lo expuesto hasta ahora, se puede afirmar que la contribución de esta investigación al conocimiento del MDO tiene lugar a varios niveles. En primer lugar, la realización de las dos pruebas llevadas a cabo para analizar el fenómeno tanto sincrónica, como diacrónicamente, ha aportado una gran cantidad de datos empíricos al respecto de la influencia que la telicidad y la afectación 
han ejercido y ejercen sobre el marcado preposicional. El hecho de haber implementado dos metodologías distintas ha provocado que los resultados producto de ambas se hayan visto reforzados y hayan arrojado una visión amplia y coherente de los factores estudiados.

Por otro lado, la revisión de la bibliografía y los datos obtenidos han permitido plantear un modelo explicativo del MDO basado en la identificación de este con la marca preposicional del dativo y en la conceptualización de ambos como una marca asociada a un grado elevado de prominencia. Esta idea de que el MDO y la marca del objeto directo son realmente producto del mismo fenómeno, aunque aparezcan con objetos que desempeñan funciones sintácticas diferentes, ya ha sido defendida por otros autores desde hace tiempo y se sigue trabajando en ella en los últimos años (Company Company 2003; 2006; 2014; Ormazábal/ Romero 2019; entre otros).

La fundamentación del modelo en las relaciones de prominencia que se generan a nivel morfosintáctico y semántico supone la apertura de una serie de caminos en la investigación de lo más sugerentes. El hecho de que los rasgos inherentes al SN sean los que determinan en primera instancia el marcado, por encima del resto de factores que se desprenden de la función que desempeña el objeto en el evento, hace pensar que, o bien existen distintos tipos de prominencia, o bien se trata siempre de un mismo tipo, pero que actúa sobre los objetos de diferente manera o a diferentes niveles. Profundizar en este aspecto puede contribuir a una mejor comprensión de las relaciones semánticas que se generan entre los diferentes participantes de un evento.

Asimismo, la conceptualización de los eventos en términos de transmisión de fuerzas, considero que también deja abierta una puerta a explorar la noción de transitividad en un sentido más amplio quizá al que se ha manejado hasta ahora. En esta línea, también queda mucho trabajo por hacer en la sistematización de la relación que existe entre los diferentes participantes de un evento y el grado de prominencia que adquieren atendiendo a su posición en la cadena causal.

De la misma forma, más allá del MDO, queda abierto el camino para continuar perfeccionando el enfoque de la dinámica de fuerzas. El objetivo ha de ser que este pueda dar cabida de una forma más minuciosa a los verbos que no implican transmisión de fuerza o a aquellos en los que no queda clara la dirección de las fuerzas que se aplican.

Para concluir se puede afirmar que la gran cantidad de datos obtenidos de las pruebas realizadas y el carácter ambicioso del enfoque implican finalmente más preguntas que certezas y dejan abiertas numerosas puertas para continuar con la investigación. 
